\title{
Relational Macros for Transfer in Reinforcement Learning
}

\author{
Lisa Torrey ${ }^{1}$, Jude Shavlik ${ }^{1}$, \\ Trevor Walker ${ }^{1}$, and Richard Maclin ${ }^{2}$ \\ 1 University of Wisconsin, Madison WI 53706, USA \\ 2 University of Minnesota, Duluth, MN 55812, USA
}

\begin{abstract}
We describe an application of inductive logic programming to transfer learning. Transfer learning is the use of knowledge learned in a source task to improve learning in a related target task. The tasks we work with are in reinforcement-learning domains. Our approach transfers relational macros, which are finite-state machines in which the transition conditions and the node actions are represented by first-order logical clauses. We use inductive logic programming to learn a macro that characterizes successful behavior in the source task, and then use the macro for decision-making in the early learning stages of the target task. Through experiments in the RoboCup simulated soccer domain, we show that Relational Macro Transfer via Demonstration (RMT-D) from a source task can provide a substantial head start in the target task.
\end{abstract}

\section{Introduction}

Knowledge transfer is an inherent aspect of human learning. When we learn to perform a task, we rarely start from scratch; instead we recall relevant knowledge from previous learning experiences and apply it. Transferring knowledge this way helps us to master new tasks more quickly.

Machine learning techniques are often designed to address isolated learning tasks. However, many machine learning domains contain multiple related tasks. Transfer learning approaches take advantage of these relationships, using knowledge learned in a source task to speed up learning in a related target task. Algorithms that allow successful transfer represent progress towards making machine learning as effective as human learning.

One area in which transfer is often desirable is reinforcement learning (RL), since standard RL algorithms can require long training times. The RL domain that we use in this work is the simulated soccer project RoboCup [9]. In Section 2 we give an overview of RL and the RoboCup domain.

Several algorithms for transfer in domains like RoboCup have been proposed, some of which we discuss in Section 3. In our own recent work [20], we introduce an approach that transfers skills using inductive logic programming (ILP), where a skill is a type of action that the RL agent uses. In this paper, we extend that approach by transferring strategies, which are action plans that may require composing several skills. We continue to use ILP to learn strategies, and we represent them with a structure that we call a relational macro. 


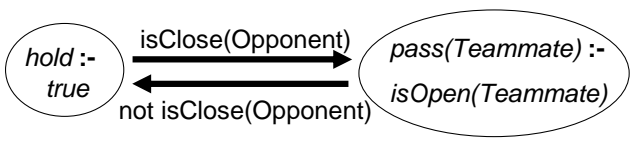

Fig. 1. A possible strategy for the RoboCup game KeepAway [13], in which the RL agent in possession of the soccer ball must execute a series of hold or pass actions to prevent its opponents from getting the ball. The rules inside nodes show how to choose actions. The labels on arcs show the conditions for taking transitions. Each node has an implied self-transition that applies by default if no exiting arc applies.

A relational macro is a finite-state machine (FSM) that uses first-order logic for decision-making. An FSM is a behavior model consisting of a set of nodes and transitions. To use a macro, an RL agent takes transitions to move between nodes representing internal states, and it chooses actions to take in each node. Its choices are determined by first-order logical clauses. Figure 1 shows a simple example of a relational macro and Section 4 provides more details on how a macro is executed.

We use inductive logic programming (ILP) to learn macros because domains like RoboCup are inherently relational. To our knowledge, fully relational RL approaches have not yet been successfully applied in domains as complex as RoboCup. However, as we showed with skill transfer, relational information can be successfully transferred between RoboCup tasks. Therefore we continue to use ILP in this approach, describing source-task behavior and relational macros in first-order logic.

Relational-macro transfer begins by examining existing source-task episodes and analyzing them to learn a successful strategy in the form of a macro. Section 5 describes our algorithm for this learning stage. There are several possible ways to use the macro to improve learning in the target task; we use it to demonstrate the successful strategy, as described in Section 6. After a short demonstration period that gives the target-task learner a head start, we continue learning the task with standard RL. We call this approach Relational Macro Transfer via Demonstration (RMT-D).

\section{Reinforcement Learning in RoboCup}

In reinforcement learning [16], an agent navigates through an environment trying to earn rewards or avoid penalties. The environment's state is described by a set of features, and the agent takes actions to cause the state to change. In one common form of RL called $Q$-learning [22], the agent learns a $Q$-function to estimate the value of taking an action from a state. An agent's policy is typically to take the action with the highest $Q$-value in the current state, except for occasional exploratory actions. After taking the action and receiving some reward (possibly zero), the agent updates its $Q$-value estimates for the current state.

Stone and Sutton [13] introduced RoboCup as an RL domain that is challenging because of its large, continuous state space and non-deterministic action 


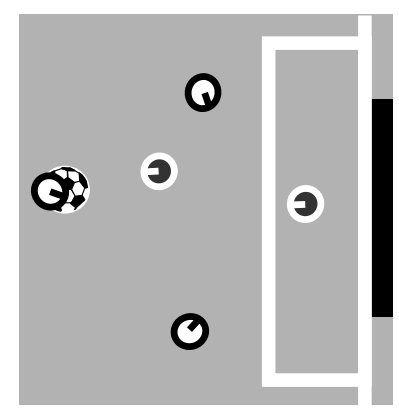

Fig. 2. Snapshot of a 3-on-2 BreakAway game. The attacking players have possession of the ball and are maneuvering against the defending team towards the goal.

effects. The RoboCup project [9] has the overall goal of producing robotic soccer teams that compete on the human level, but it also has a software simulator for research purposes. Since the full game of soccer is quite complex, researchers have developed several simpler games within the RoboCup simulator. See Figure 2 for a snapshot of one of these games.

In $M$-on- $N$ BreakAway [21], the objective of the $M$ reinforcement learners called attackers is to score a goal against $N-1$ hand-coded defenders and a goalie. The game ends when they succeed, when an opponent takes the ball, when the ball goes out of bounds, or after a time limit of 10 seconds. The learners receive a +1 reward if they score a goal and 0 reward otherwise. The attacker who has the ball may choose to move (ahead, away, left, or right with respect to the goal center), pass to a teammate, or shoot (at the left, right, or center part of the goal).

RoboCup tasks are inherently multi-agent games, but a standard simplification is to have only one learning agent. This agent controls the attacker currently in possession of the ball, switching its "consciousness" between attackers as the ball is passed. Attackers without the ball follow simple hand-coded policies that position them to receive passes.

Table 1 shows the state features for BreakAway, which mainly consist of distances and angles between players and the goal. They are represented in logical notation, though our RL algorithm uses the grounded versions of these predicates in a fixed-length feature vector. Capitalized atoms indicate typed variables, while constants and predicates are uncapitalized. The attackers (labeled $a 0, a 1$, etc.) are ordered by their distance to the agent in possession of the ball $(a 0)$, as are the non-goalie defenders $(d 0, d 1$, etc.).

Our RL implementation uses a $S A R S A(\lambda)$ variant of $Q$-learning [15] and employs a support vector machine for function approximation [7]. We relearn the $Q$-function using the SVM after every batch of 25 games. The exploration rate begins at $2.5 \%$ and decays exponentially over time. Stone and Sutton [13] found that discretizing the continuous features into Boolean interval features called tiles is useful for learning in RoboCup; following this approach we create 32 tiles per feature. 
Table 1. The features that describe a BreakAway state.

\begin{tabular}{ll}
\hline distBetween(a0, Player) & distBetween(Attacker, goalCenter) \\
distBetween(Attacker, ClosestDefender) & distBetween(a0, GoalPart) \\
angleDefinedBy(Attacker, a0, ClosestDefender) & timeLeft \\
angleDefinedBy(topRight, goalCenter, a0) & distBetween(Attacker, goalie) \\
angleDefinedBy(GoalPart, a0, goalie) & angleDefinedBy(Attacker, a0, goalie) \\
\hline
\end{tabular}

Agents in the games of 2-on-1, 3-on-2, and 4-on-3 BreakAway take approximately 3000 training episodes to reach a performance asymptote in our system. These three games are similar, but their differences in the numbers of attackers and defenders cause substantial differences in their optimal policies. The largest difference is between 2-on-1 and the others, since there are no non-goalie defenders in 2-on-1 BreakAway. Despite the differences, the tasks do have the same objective and can be expected to require similar strategies, which makes relational macros an attractive technique for transferring between them.

\section{Related Work in Transfer Learning}

The goal in transfer learning is to speed up learning in a target task by transferring knowledge from a related source task. One straightforward way to do this in reinforcement learning is to begin performing the target task using the learned source-task models. Taylor et al. [19] use this type of transfer method, which we refer to as model reuse.

Another approach that has been proposed is to follow source-task policies during the exploration steps of normal RL in the target task, instead of doing random exploration. This approach is referred to as policy reuse and is performed by Fernandez and Veloso [5].

Our previous work includes a method called skill transfer [20]. In skill transfer, we learn rules with ILP that indicate when the agent chooses to take a single source-task action. There are multiple ways that these skills could be used in the target task; we use an advice-taking approach in this previous work. Our advice places soft constraints on the target-task solution that can be followed or ignored according to how successful they are. Taylor and Stone [18] also learn a set of rules for taking actions, and they propose different advice-taking mechanisms: for example, they give a $Q$-value bonus to the advised action.

There are also approaches for transferring multi-step action sequences, such as those of Perkins and Precup [10] and Soni and Singh [11]. Known as options, these sequences have their own internal $Q$-functions that are followed until they reach a termination state. The target-task learner treats options as alternative actions. Croonenborghs et al. [1] learn relational options for use in relational reinforcement learning (RRL). Options are often used in hierarchical RL [2] as well as in transfer learning. 
Relational reinforcement learning [17] itself is a related topic. In RRL, state descriptions and learned models use first-order logic, which clearly provides opportunities for transferring concepts in first-order logic. Driessens et al. [4] and Stracuzzi and Asgharbeygi [14] point out some of these opportunities.

We propose to perform transfer by learning relational macros and using them to demonstrate successful behavior in the target task. Our approach is related to several of the methods described above. It could be viewed as a type of model reuse or policy reuse that creates an abstract version of the source-task model instead of reusing it directly. Like skill transfer it uses ILP, but it involves multi-step strategies instead of single actions. It shares the idea of transferring sub-policies with option transfer, but an option traditionally represents a single policy while a macro contains a separate sub-policy at each node.

\section{Executing a Relational Macro}

We have defined a relational macro as a finite-state machine [6]. An FSM models the behavior of a system in the form of a directed graph. The nodes of the graph represent states of the system, and in our case they represent internal states of the agent in which different policies apply.

The policy of a node can be to take a single action, such as move(ahead) or shoot(goalLeft), or to choose from a class of actions, such as pass(Teammate). In the latter case a node has first-order logical clauses to decide which grounded action to choose. An FSM begins in a start node and has conditions for transitioning between nodes. In a relational macro, these conditions are also sets of first-order logical clauses.

We refer again to the example macro in Figure 1. When executing this macro, a KeepAway agent begins in the initial node on the left. The only action it can choose in this node is hold. It remains there, taking the default self-transition, until the condition isClose(Opponent) becomes true for some opponent player. Then it transitions to the second node, where it evaluates the pass(Teammate) rule to choose an action. If the rule is true for just one teammate player, it passes to that teammate; if several teammates qualify, it randomly chooses between them; if no teammate qualifies, it abandons the macro and reverts to using the $Q$-function to choose actions. The receiving teammate then becomes the learning agent, and it remains in the pass node if an opponent is close or transitions back to the hold node otherwise.

Figure 1 is a simplification in one respect: each transition and node in a macro has an entire set of rules, rather than just one rule. This allows us to represent disjunctive conditions. When more than one grounded action or transition is possible (when multiple rules match), the agent obeys the rule that has the highest score. The score of a rule is the estimated probability that following it will lead to a successful game. We estimate these probability scores from sourcetask data. 


\section{Learning a Relational Macro}

We learn a macro by analyzing source-task data. We assume that this data is available because we have previously learned the source task and stored the games generated during the learning process. The method by which the source task was learned is not particularly important, since the data we use only consists of states, actions, and rewards. However, it is important that the data include source-task games from early in the learning curve as well as later, so that there are examples of games that do not attempt to use the final learned strategy. In our system we include all 3000 games from the source-task learning curve.

Given this data, we use inductive logic programming (ILP) to characterize successful behavior in the source task. Specifically, we use a locally modified version of Aleph [12]. The Aleph algorithm selects an example, builds the most specific clause that entails the example within the provided language restrictions, and searches for a more general clause that maximizes a provided scoring function.

The precision of a rule is the fraction of examples it calls positive that are truly positive, and the recall is the fraction of truly positive examples that it correctly calls positive. The scoring function we use is

$$
F(1)=\frac{2 * \text { Precision } * \text { Recall }}{\text { Precision }+ \text { Recall }}
$$

because we consider both precision and recall to be important. We use both the heuristic and randomized search algorithms provided by Aleph.

Recall that a macro consists of a set of nodes along with rulesets for transitions and action choices. The simplest algorithm for learning a macro might be to provide Aleph with language restrictions that allow it to learn both the structure and the rulesets simultaneously. However, this would be a very large search space. To make the search more feasible, we separate it into two phases: first we learn the structure, and then we learn each ruleset independently. Each phase therefore has its own language restrictions, which we detail in the following sections. The overall algorithm is summarized in Table 2.

Note that one final step might be necessary if the actions and features in the source and target tasks are not identically named: a mapping from source-task names to target-task names, as in Torrey et al. [20,21]. Our approach does not even require the tasks to be completely isomorphic, because we can set the Aleph language restrictions so that only source-task elements that have corresponding target-task elements appear in the macro.

\subsection{Structure Learning}

The first phase in our RMT-D algorithm for learning a macro is the structurelearning phase. The objective is to find a sequence of actions that distinguishes successful games from unsuccessful games. The sequence does not need to separate the games perfectly, and indeed we should not expect it to, because it does 
Table 2. Our RMT-D algorithm for learning a relational macro from a source task.

Phase 1: Structure learning

Collect games from source task

Let $P o s=$ high-reward games

Let $N e g=$ low-reward games

Learn a macro sequence that distinguishes Pos from $\mathrm{Neg}$

Phase 2: Ruleset learning

Collect games $G_{\text {good }}$ that contain the macro sequence and are high-reward

Collect games $G_{b a d}$ that are low-reward

For each node $\mathrm{N}$ in the macro sequence

For each action A represented by node $\mathrm{N}$

Let $P o s=G_{\text {good }}$ states from node $\mathrm{N}$ that took action A

Let $N e g=G_{\text {good }}$ states from node $\mathrm{N}$ that took action B $\neq \mathrm{A}$

$\cup G_{b a d}$ states that ended with action A

Learn a ruleset that distinguishes Pos from $\mathrm{Neg}$

For each transition $\mathrm{T}$ in the macro

Let $P o s=G_{\text {good }}$ states that took transition T

Let $N e g=G_{\text {good }}$ states that could have taken transition $\mathrm{T}$ and did not

$\cup G_{b a d}$ states that ended with transition $\mathrm{T}$

Learn a ruleset that distinguishes Pos from $\mathrm{Neg}$

not yet have any conditions on states. The structure only needs to provide a good starting point for the second phase.

The language restrictions for Aleph in this phase are as follows. Let the predicate action Taken $\left(G, S_{1}, A, P, S_{2}\right)$ denote that action $A$ with argument $P$ was taken in game $G$ at step $S_{1}$ and repeated until step $S_{2}$. Aleph must construct a clause macroSequence $(G)$ with a body that contains a combination of these predicates. The first predicate may introduce two new variables, $S_{1}$ and $S_{2}$, but the rest must use an existing variable for $S_{1}$ while introducing another new variable $S_{2}$. In this way Aleph finds a connected sequence of actions that translates directly to a linear node structure.

We provide Aleph with sets of positive and negative examples, where positives are games with high overall reward and negatives are those with low overall reward. For BreakAway, this is a straightforward separation of scoring and nonscoring games. For tasks with more continuous rewards, we could set thresholds or upper and lower percentiles on the overall reward acquired during a game.

We store all the clauses that Aleph encounters during its search that separate the positive and negative examples with at least 50\% accuracy. After the heuristic and randomized searches finish, we take the sequence with the highest $\mathrm{F}(1)$ score as the macro structure.

For instance, suppose that the scoring BreakAway games consistently look like these examples:

Game 1: move(ahead), pass(a1), shoot(goalRight)

Game 2: move(ahead), move(ahead), pass(a2), shoot(goalLeft) 


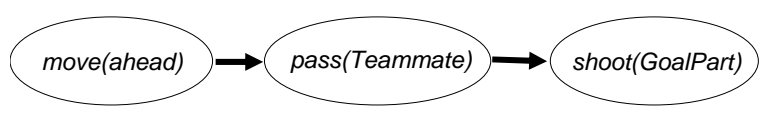

Fig. 3. The structure that corresponds to the example macro clause in Section 5.1.

Assuming that the non-scoring games have different patterns than the examples above do, Aleph might learn the following clause to characterize a scoring game:

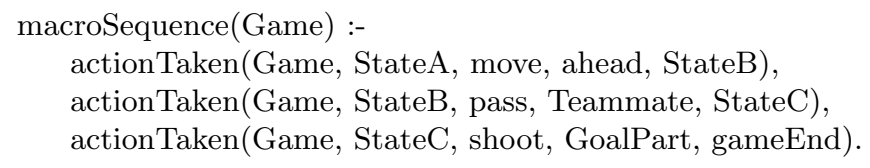

The macro structure corresponding to this sequence is shown in Figure 3. The policy in the first node will be to take a single action, move(ahead). In the second node the policy will be to consider multiple pass actions, and in the third node the policy will be to consider multiple shoot actions. The conditions for choosing an action, and for taking transitions between nodes, are learned in the next phase.

\subsection{Ruleset Learning}

The second phase in our RMT-D algorithm for learning a macro is the rulesetlearning phase. The objective is to describe when transitions and actions should be taken based on the RL state features. We learn a ruleset for each transition and each action independently, so that we perform several smaller, in-depth seaches rather than one large search. Because of this, variables in these rules are local to a node rather than global to the entire macro.

The language restrictions for Aleph in this phase are as follows. There is one predicate for each state feature of the RL task (for BreakAway, these are in Table 1). To describe the conditions on state $S$ under which a transition should be taken, Aleph must construct a clause transition $(S)$ with a body that contains a combination of these predicates. To describe the conditions under which an action should be taken, Aleph must construct a clause action(S, Action, ActionArg).

Aleph may learn some action rules in which the action argument is grounded, such as action (S, move, ahead), as well as rules in which the action argument remains a variable, such as action(S, pass, Teammate). In the case of the move action in BreakAway the action argument in a rule is always grounded, since the original state features do not include useful references to move directions. We could have defined additional predicates that did, but we chose to use only the original features. Note that it is still possible to have a state move(Direction) for taking multiple move actions, but the rules for choosing a grounded move action will use only grounded arguments. 


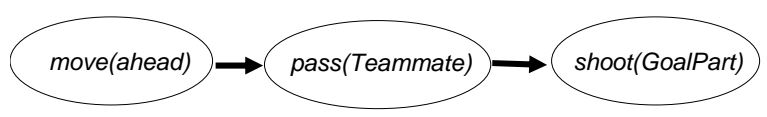

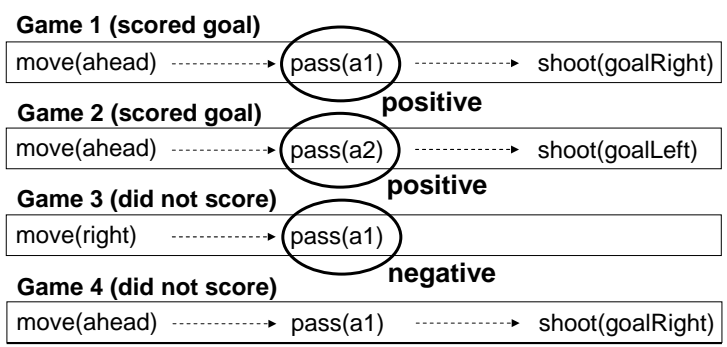

Fig. 4. Training examples (states circled) for pass(Teammate) rules in the second node of the pictured macro. The pass states in Games 1 and 2 are positive examples. The pass state in Game 3 is a negative example; this game did not follow the macro, but the pass action led directly to a negative game outcome. The pass state in Game 4 is not an unambiguous example because a later action may have been responsible for the bad outcome.

We provide Aleph with sets of positive and negative examples, consisting of states in source-task games that took the transition or action. Consider the macro structure in Figure 3; we will describe the action datasets for the pass node and the transition datasets for the transition from the move node to the pass node. Let $G_{\text {good }}$ represent the set of high-reward source-task games that contain the macro sequence and let $G_{b a d}$ represent the set of low-reward source-task games.

In the action datasets for the pass node, the positive examples are states in $G_{\text {good }}$ games that fall into that node. The negative examples are states in $G_{b a d}$ games in which the last step of the unsuccessful game was the node action, pass. This indicates that the pass action led directly to a negative game outcome. Figure 4 illustrates some hypothetical action-choice examples.

In the transition datasets for the transition from the move node to the pass node, the positive examples are states in $G_{\text {good }}$ games that match the pass node and for which the previous state matched the move node. A negative example is a state in a $G_{\text {good }}$ game that does not match the pass node even though the previous state matched the move node. Other negative examples are states in $G_{b a d}$ games in which the last step of the unsuccessful game was a transition from the move node to the pass node. Figure 5 illustrates some hypothetical transition examples.

As in the first phase, we store all the clauses that Aleph encounters during the search that classify the training data with at least $50 \%$ accuracy. However, instead of selecting a single best clause as we did in the previous phase, we collect from these a ruleset for each transition and each action. We wish to have one strategy (i.e. one finite-state machine), but there may be multiple reasons for making internal choices. 

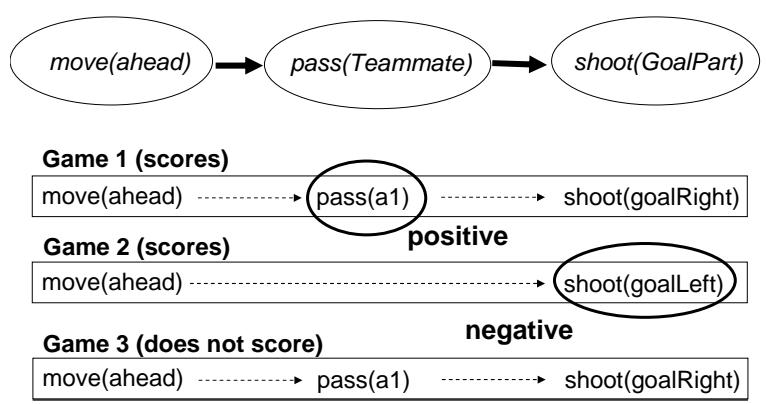

Fig. 5. Training examples (states circled) for the transition from move to pass in the pictured macro. The pass state in Game 1 is a positive example. The shoot state in Game 2 is a negative example; the game began by following the macro but did not take the transition from move to pass. The pass state in Game 3 is not an unambiguous example because a later step may have been responsible for the bad outcome.

Our procedure for greedily selecting which clauses are included in a ruleset is summarized in Table 3. We sort the rules by decreasing precision and walk through the list, adding rules to the final ruleset if they increase the set's recall and do not decrease its $\mathrm{F}(1)$ score.

We assign each rule a score that may be used to decide which rule to obey if multiple rules match while executing the macro. The score is an estimate of the probability that following the rule will lead to a successful game. We determine this estimate by collecting training-set games that followed the rule and calculating the fraction of these that ended successfully.

\section{Transferring a Relational Macro}

A relational macro describes a strategy that was successful in the source task. There are several ways we could use this information to improve learning in a related target task. One possibility is to treat it as advice [20], as we did in skill transfer, putting soft constraints on the $Q$-learner that influence its solution. The benefit of this approach is its robustness to error: if the source-task knowledge is less appropriate to the target task than the user expected, the target-task agent can learn to disregard the soft constraints, avoiding negative transfer effects.

On the other hand, the advice-taking approach is conservative and can be somewhat slow to reach its full effect, even when the source-task knowledge is highly appropriate to the target task. Since a macro is a full strategy rather than isolated skills, we might achieve good target-task performance more quickly by demonstrating the strategy in the target task and using it as a starting point for learning. This is a more aggressive approach, carrying more risk for negative transfer if the source and target tasks are not similar enough. Still, if the user believes that the tasks are similar enough, the potential benefits could outweigh that risk. 
Table 3. The RMT-D procedure for selecting the final ruleset for one transition or action. Rules are added to the final set if they cover previously uncovered positive examples and do not decrease the overall score. The scoring function is the $\mathrm{F}(1)$ measure.

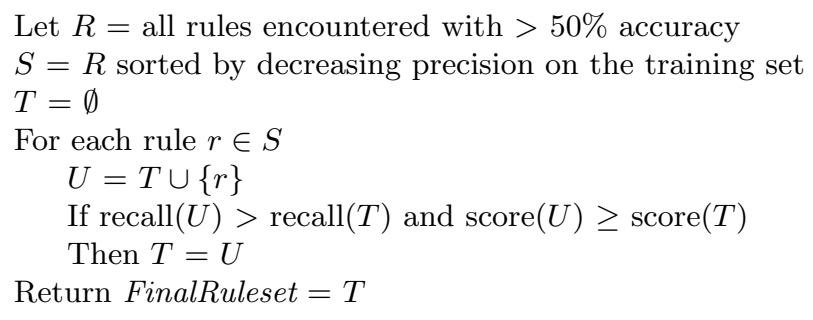

There are intermediate approaches with more moderate benefits and risks, such as using the macro as an option. Here we take full advantage of the potential benefits, and also provide a contrasting method to skill transfer, by presenting the more aggressive demonstration method.

Our target-task learner therefore begins by simply executing the macro strategy for a set of episodes, instead of exploring randomly as an untrained RL agent would traditionally do. In this demonstration period, we generate examples of $Q$-values: each time the macro chooses an action because a high-scoring rule matched, we use the rule score to estimate the $Q$-value of the action. Recall the the rule score is the estimated probability that following the rule leads to a successful game. Since BreakAway has $Q$-values ranging from zero to one, we simply set the estimate equal to the rule score (if this were not the case, we could multiply the probability by an appropriate scaling factor to fit a larger $Q$-value range). We also use rule scores to produce $Q$-value estimates for other actions for which rules fired. Finally, we infer that actions for which no rules fired had very low $Q$-values, which in the BreakAway domain we estimate as zero.

Note that the examples with low estimated $Q$-values are necessary to ensure that the initial $Q$-function is not overly optimistic in unexplored areas. Driessens and Dzeroski also encountered this problem in their work on guidance in RRL; they addressed it by interleaving imitation with exploration [3].

For each step of the demonstration we therefore have a $Q$-value estimate for each action, and via support vector regression we use these to learn an initial $Q$-function for the target task. The demonstration period lasts for 100 games in our system, and as usual after each batch of 25 games we relearn the $Q$ function. After 100 games, we continue learning the target task with standard $\mathrm{RL}$. This generates new $Q$-value examples in the standard way, and we combine these with the old macro-generated examples as we continue relearning the $Q$ function after each batch. As the new examples accumulate, we gradually drop the old examples by randomly removing them at the rate that new ones are being added. 
Since standard RL has to act mostly randomly in the early steps of a task, a good macro strategy can provide a large immediate advantage. The performance level of the demonstrated strategy is unlikely to be as high as the target-task agent can achieve with further training, unless the tasks are similar enough to make transfer a trivial problem, but the hope is that the learner can smoothly improve its performance from the level of the demonstration up to its asymptote. If there is limited time and the target task cannot be trained to its asymptote, then the immediate advantage that macros can provide may be even more valuable in comparison to methods like skill transfer.

\section{$7 \quad$ Experimental Results}

We present results from transfer experiments with RMT-D in the RoboCup domain. To test our approach, we learn a macro from data acquired while training 2-on-1 BreakAway and transfer it to both 3-on-2 and 4-on-3 BreakAway. We learn the source task with standard RL for 3000 games, and then we train the target tasks for 3000 games to show both the initial advantage of the macros and the behavior as training continues.

The macros that RMT-D learned from the five source runs all had similar structures. The most common version is shown in Figure 6. In one of the runs the initial pass node was not included, and the ordering of shoot(goalRight) and shoot (goalLeft) varied, as would be expected in the symmetrical BreakAway domain. The presence of two shoot nodes may seem counterintuitive, but it appears that the RL agent uses the first shot as a feint to lure the goalie in one direction, counting on a teammate to intercept the shot before it reaches the goal. When it does, the learning agent switches to the teammate in possession of the ball and performs the second shot, which is actually intended to score. This tendency of RL agents to use actions in unintended ways is an indication of the difficulties that can arise when learning relational concepts from RL data.

Figures 7 and 8 show our results in 3-on-2 and 4-on-3 BreakAway respectively. We compare our approach against $Q$-learning as well as two related transfer methods: model reuse [19] and skill transfer [20]. Each curve in the figure is an average of 25 runs and has points smoothed over the previous 500 games to smooth over the high variance in the RoboCup domain. For the transfer algorithms, there are five target-task runs generated from each of five sourcetask runs, to allow for variance in both stages of learning.

Our agents in 2-on-1 BreakAway reach a performance asymptote of scoring in approximately $70 \%$ of the episodes. The macros learned from the 2 -on- 1 source

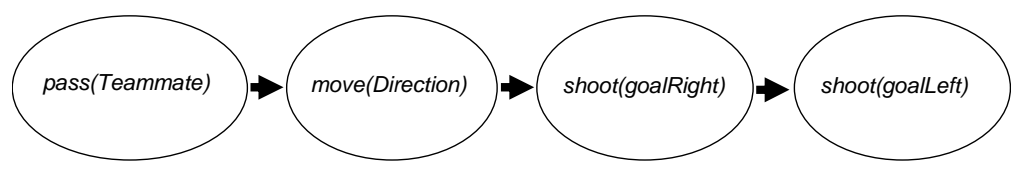

Fig. 6. One of the five macro structures learned from 2-on-1 BreakAway runs. There are between 10 and 20 rules associated with each transition and action, so those are not shown. 


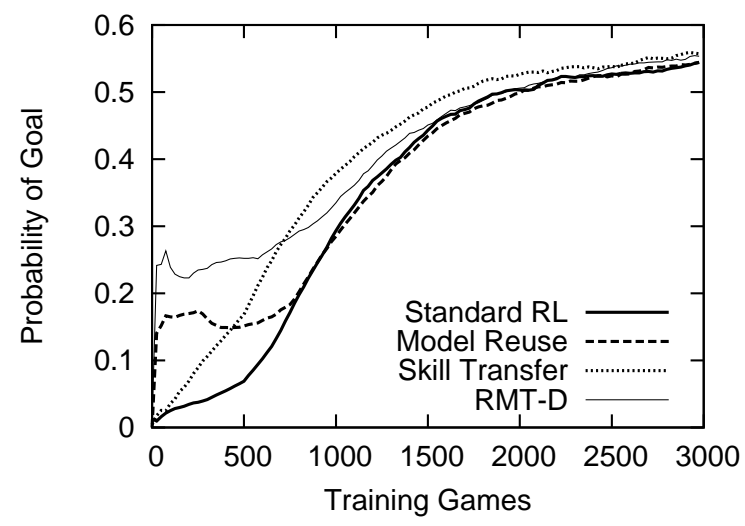

Fig. 7. Probability of scoring a goal in 3-on-2 BreakAway, with Q-learning and with three transfer approaches that use 2-on-1 BreakAway as the source task.

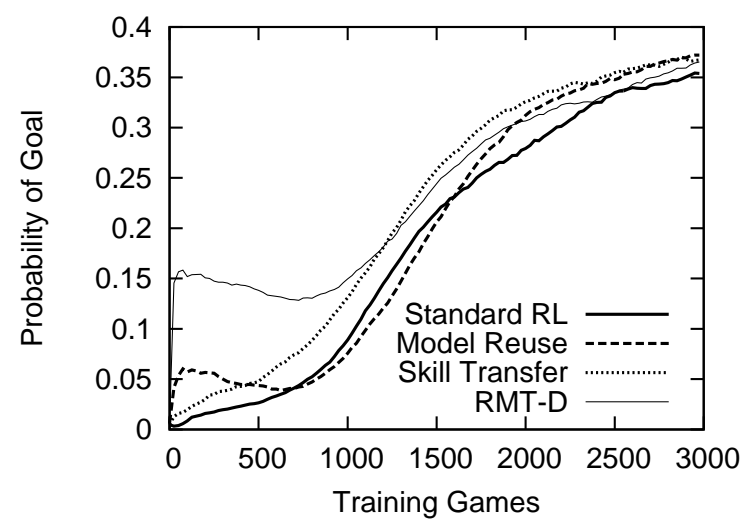

Fig. 8. Probability of scoring a goal in 4-on-3 BreakAway, with $Q$-learning and with three transfer approaches that use 2-on-1 BreakAway as the source task.

runs, when executed in 2-on-1 BreakAway, score in approximately $50 \%$ of the episodes. (A random policy scores in less than 1\% of the episodes.) The macros therefore appear to capture the majority of the successful behavior of the source task, though they do not describe it completely. Capturing source-task behavior more completely, while avoiding overfitting, is one topic for future work.

All of the transfer algorithms speed up learning in comparison to $Q$-learning, but the benefits they provide are different. Model reuse and relational macros both provide an advantage in the early performance of the target-task learner. RMT-D produces a larger advantage in these scenarios than model reuse does, and it scales better as the distance between the source and target grows. Skill transfer provides no initial benefit, but then develops a steady advantage over $Q$-learning. During the middle section of the learning curve it performs slightly better than RMT-D before they all converge at the asymptote.

In pointwise $t$-test comparisons at the $99 \%$ confidence level, the RMT-D curve is significantly above the model-reuse curve for the first 1100 episodes in 
Figure 7 and 1425 episodes in Figure 8. The RMT-D curve is significantly above the skill-transfer curve for the first 575 episodes in Figure 7 and 875 episodes in Figure 8. The skill-transfer curve is significantly above the RMT-D curve at just one point in Figure 7 (at 1825 episodes) and never in Figure 8, and the model-reuse curve is never significantly above the RMT-D curve in either figure.

We also tried an algorithm that combines skill transfer via advice with RMTD. The combination is straightforward: we begin by demonstrating the macro as in RMT-D, and we incorporate advice when learning the $Q$-function as in skill transfer. This produces a learning curve (not shown) that is not significantly different from the RMT-D curve. The substantial early effects of transferring a macro via demonstration apparently overwhelm the effects of skill-transfer advice.

\section{Conclusions and Future Work}

Knowledge transfer in reinforcement learning is an interesting and challenging problem, and inductive logic programming is a powerful tool to apply to it. The use of ILP allows us to transfer the kind of information that humans might transfer: strategies with decisions in first-order logic. We describe an approach for transferring relational macros from a source task that gives the target-task learner a significant head start. Our approach produces consistently higher initial performance than standard RL and several related transfer methods.

In future work, we plan to investigate alternative macro designs that may capture the source-task behavior more completely. While a single linear action sequence appears to explain the majority of our agents' success in the source task, other configurations might perform better. We are interested in trying a statistical relational learning (SRL) approach to estimate probabilities and to make decisions from rulesets.

Our RMT-D algorithm is most effective when the user is confident that the source-task strategy is a reasonable approximation of a good target-task strategy. However, relational macros might be applicable in more distant transfer scenarios, such as when only part of a source-task strategy is useful in a target task. We plan to investigate alternative ways to apply relational macros in the target task to make this possible. Potential frameworks for this include options [1] and advice-taking [8]. We are also interested in incorporating human advice into relational structures.

Another direction for future work is the refinement of relational macros during target-task learning. The parameters or structure of a macro could be updated based on early experience in the target task. This is a problem of theory refinement, which is an area of interest for transfer learning.

\section{Acknowledgements}

This research is supported by DARPA IPTO grants HR0011-04-1-0007 and FA8650-06-C-7606. 


\section{References}

1. T. Croonenborghs, K. Driessens, and M. Bruynooghe. Learning relational skills for inductive transfer in relational reinforcement learning. In International Conference on Inductive Logic Programming, 2007.

2. T. Dietterich. Hierarchical reinforcement learning with the MAXQ value function decomposition. Journal of Artificial Intelligence Research, 13:227-303, 2000.

3. K. Driessens and S. Dzeroski. Integrating guidance into relational reinforcement learning. Machine Learning, 57(3):271-304, 2004.

4. K. Driessens, J. Ramon, and T. Croonenborghs. Transfer learning for reinforcement learning through goal and policy parametrization. In ICML Workshop on Structural Knowledge Transfer for Machine Learning, 2006.

5. F. Fernandez and M. Veloso. Policy reuse for transfer learning across tasks with different state and action spaces. In ICML Workshop on Structural Knowledge Transfer for Machine Learning, 2006.

6. A. Gill. Introduction to the Theory of Finite-state Machines. McGraw-Hill, 1962.

7. R. Maclin, J. Shavlik, L. Torrey, and T. Walker. Knowledge-based support vector regression for reinforcement learning. In IJCAI Workshop on Reasoning, Representation, and Learning in Computer Games, 2005.

8. R. Maclin, J. Shavlik, L. Torrey, T. Walker, and E. Wild. Giving advice about preferred actions to reinforcement learners via knowledge-based kernel regression. In AAAI Conference on Artificial Intelligence, 2005.

9. I. Noda, H. Matsubara, K. Hiraki, and I. Frank. Soccer server: A tool for research on multiagent systems. Applied Artificial Intelligence, 12:233-250, 1998.

10. T. Perkins and D. Precup. Using options for knowledge transfer in reinforcement learning. Technical Report UM-CS-1999-034, 1999.

11. V. Soni and S. Singh. Using homomorphisms to transfer options across continuous reinforcement learning domains. In AAAI Conference on Artificial Intelligence, 2006.

12. A. Srinivasan. The Aleph manual, 2001.

13. P. Stone and R. Sutton. Scaling reinforcement learning toward RoboCup soccer. In International Conference on Machine Learning, 2001.

14. D. Stracuzzi and N. Asgharbeygi. Transfer of knowledge structures with relational temporal difference learning. In ICML Workshop on Structural Knowledge Transfer for Machine Learning, 2006.

15. R. Sutton. Learning to predict by the methods of temporal differences. Machine Learning 3, pages 9-44, 1988.

16. R. Sutton and A. Barto. Reinforcement Learning: An Introduction. MIT Press, 1998.

17. P. Tadepalli, R. Givan, and K. Driessens. Relational reinforcement learning: An overview. In ICML Workshop on Relational Reinforcement Learning, 2004.

18. M. Taylor and P. Stone. Cross-domain transfer for reinforcement learning. In International Conference on Machine Learning, 2007.

19. M. Taylor, P. Stone, and Y. Liu. Value functions for RL-based behavior transfer: A comparative study. In AAAI Conference on Artificial Intelligence, 2005.

20. L. Torrey, J. Shavlik, T. Walker, and R. Maclin. Skill acquisition via transfer learning and advice taking. In ECML, 2006.

21. L. Torrey, T. Walker, J. Shavlik, and R. Maclin. Using advice to transfer knowledge acquired in one reinforcement learning task to another. In $E C M L, 2005$.

22. C. Watkins. Learning from delayed rewards. PhD thesis, University of Cambridge, 1989. 